\title{
Visceral Leishmaniasis with Associated Common, Uncommon, and Atypical Morphological Features on Bone Marrow Aspirate Cytology in Nonendemic Region
}

\author{
Harish Chandra, ${ }^{1}$ Smita Chandra, ${ }^{1}$ and Rajeev Mohan Kaushik ${ }^{2}$ \\ ${ }^{1}$ Department of Pathology, Himalayan Institute of Medical Sciences, Swami Ram Nagar, Doiwala, \\ Dehradun, Uttarakhand 248140, India \\ ${ }^{2}$ Department of Medicine, Himalayan Institute of Medical Sciences, Swami Ram Nagar, Doiwala, \\ Dehradun, Uttarakhand 248140, India
}

Correspondence should be addressed to Harish Chandra; drharishbudakoti31@yahoo.co.in

Received 25 April 2013; Revised 16 July 2013; Accepted 7 August 2013

Academic Editor: Luis E Cuevas

Copyright (C) 2013 Harish Chandra et al. This is an open access article distributed under the Creative Commons Attribution License, which permits unrestricted use, distribution, and reproduction in any medium, provided the original work is properly cited.

Objectives. The present study was conducted to categorise the morphological features on bone marrow aspirate cytology into common, uncommon, and atypical features in a nonendemic region which would be helpful in clinching an early and correct diagnosis especially in clinically unsuspected cases. Methods. The morphological features on bone marrow were categorized into common, uncommon, and atypical in cases of leishmaniasis from non endemic region. Results. Out of total 27 cases, $77.7 \%$ were residents of places at the height of $500 \mathrm{~m}$ or above and fever was the most common presentation followed by hepatosplenomegaly. Plasmacytosis, hemophagocytosis were the common cytological features while dysmyelopoiesis, presence of leishmania bodies in nonhistiocytic cells, and granuloma with necrosis were uncommon features. Aggregates of LD bodies in form of ring, floret, or strap shapes along with giant cells constitute the atypical morphological features. Conclusion. The knowledge of common, uncommon, and atypical features on bone marrow aspirate cytology is helpful in clinching an early and correct diagnosis of leishmaniasis especially in non endemic areas where clinical suspicion is low. These features will guide the pathologist for vigilant search of LD bodies in the marrow for definite diagnosis and thus will also be helpful in preventing unnecessary workups.

\section{Introduction}

Visceral leishmaniasis (VL) is vector borne parasitic disease which results from the infection of macrophages in reticuloendothelial system associated with immunoinflammatory response [1]. The higher altitudes negatively affect the distribution of vector, and therefore leishmaniasis is considered to be almost absent in highlands [2]. However, cases demonstrating Leishmania donovani (LD) bodies on bone marrow aspirate cytology have been observed in our tertiary care center which caters to nonendemic hilly and subhilly regions. Although bone marrow findings in VL have been described in the literature, studies have rarely categorized these morphological features into common, uncommon, and atypical features according to their frequency and presentation [3-5]. Therefore the present study was conducted to evaluate the clinicohematological profile and study the morphological features on bone marrow examination in cases demonstrating LD bodies in marrow aspirate along with the categorization of these morphological features into common, uncommon, and atypical features. It was also intended to study whether these common, uncommon, and atypical morphological features observed in bone marrow in this nonendemic region differed from prior limited studies that graded the morphological features $[3,4]$. This knowledge of morphological categorization would be helpful in clinching an early and correct diagnosis in nonendemic areas especially in clinically unsuspected cases and may prevent the use of advanced and costly diagnostic modalities along with unnecessary workups.

\section{Material and Methods}

The study was conducted in the Pathology department of the institute which included all the cases of leishmaniasis 
TABLE 1: Clinical presentation in cases showing Leishmania donovani amastigotes in bone marrow.

\begin{tabular}{lcc}
\hline Clinical presentation & $\begin{array}{c}\text { Number } \\
\text { of cases }\end{array}$ & $\begin{array}{c}\text { Percentage } \\
(\%)\end{array}$ \\
\hline Fever & 24 & 88.8 \\
Hepatosplenomegaly & 18 & 66.6 \\
Loss of weight, appetite, and lethargy & 08 & 29.6 \\
Nausea and vomiting & 07 & 25.9 \\
Cough with expectoration & 05 & 18.5 \\
Lymphadenopathy & 03 & 11.1 \\
Diarrhoea & 03 & 11.1 \\
Generalized anasarca & 03 & 11.1 \\
Epistaxis and bleeding per rectum & 03 & 11.1 \\
Miscellaneous (jaundice and joint pains) & 03 & 11.1 \\
\hline
\end{tabular}

which were diagnosed on bone marrow aspirate cytology by demonstration of LD bodies on Giemsa stained smears over a period of six and half years. Relevant clinical details, investigations, associated morphological features on bone marrow aspirate and trephine biopsy (wherever it was performed), and residential address along with history of visit to endemic regions were recorded for every case. The morphological findings observed on bone marrow aspirate cytology and trephine biopsy were reviewed by two pathologists and were categorized into common, uncommon, and atypical according to the frequency of presentation and as described by prior studies in the literature $[3,4]$. The morphological findings were also compared with previous studies especially by Daneshbod et al. which was done in endemic area so as to assess any morphological differences depending on endemicity [4]. Hemophagocytosis (HPS) was graded on aspirate smears as 0 -absent; $(1+)$ (mild) $<2$, histiocytes with HPS/slide; (2+) (moderate) 2-5 histiocytes with HPS/slide; and $(3+)$ (severe) $>5$ histiocytes with HPS/slide, and average parasite density (APD) was also graded on smears as described in the literature [3].

\section{Results}

The study included total 27 cases of visceral leishmaniasis which were diagnosed on bone marrow aspirate cytology over period of six and half years. Out of these, 22 cases were observed in males and 5 cases in females, and all the cases were of nonendemic region. 18 cases were found in people who were either labourers or farmers or did cattle rearing while 9 cases were employed in various jobs in banks, hospitals, and business or were students. Out of the total 27 cases, $77.7 \%$ ( 21 cases) were residents of places which are at the height of $500 \mathrm{~m}$ or above sea level and 8 cases were from $1500 \mathrm{~m}$ or above sea level.

Out of the total 27 cases, 22 cases were clinically unsuspected, one case each was associated with human immunodeficiency virus (HIV), tuberculosis, rheumatoid arthritis, and two cases were associated with typhoid. Table 1 shows the clinical presentation of cases with LD bodies in bone marrow.
TABle 2: Common hematological and bone marrow findings observed in leishmaniasis.

\begin{tabular}{lcc}
\hline & $\begin{array}{c}\text { Number } \\
\text { of cases }\end{array}$ & $\begin{array}{c}\text { Percentage } \\
\text { of cases (\%) }\end{array}$ \\
\hline Peripheral blood smear & 26 & 96.2 \\
Pancytopenia & 27 & 100 \\
Anemia & 26 & 96.2 \\
Leucopenia & 26 & 96.2 \\
Thrombocytopenia & 25 & 92.5 \\
Others (agglutination, fragmented RBC, or & & \\
Rouleaux formation) & & \\
Bone marrow aspirate cytology & 27 & 100 \\
Increased histiocytes & 26 & 96.2 \\
Plasmacytosis & 22 & 81.4 \\
Erythroid hyperplasia & & \\
Distribution of LD bodies & 00 & 00 \\
$\quad$ Intrahistiocytic only & 01 & 3.7 \\
$\quad$ Extrahistiocytic only & 26 & 96.2 \\
$\quad$ Mixed & 19 & 70.3 \\
Hemophagocytosis (mild to moderate) & 24 & 88.8 \\
Average parasite density (1-2+) & & \\
Bone marrow biopsy (total 19 cases) & 05 & 26 \\
Increased cellularity & 06 & 31.5 \\
Increased vascularity & & \\
\hline
\end{tabular}

TABLE 3: Uncommon hematological and bone marrow findings observed in leishmaniasis.

\begin{tabular}{lcc}
\hline & $\begin{array}{c}\text { Number } \\
\text { of cases }\end{array}$ & $\begin{array}{c}\text { Percentage } \\
\text { of cases (\%) }\end{array}$ \\
\hline $\begin{array}{l}\text { Peripheral blood smear } \\
\text { Monocytosis }\end{array}$ & 05 & 18.5 \\
Nucleated RBC & 06 & 22.2 \\
Bone marrow aspirate cytology & & \\
Plasma cells with abnormal inclusions & 04 & 14.8 \\
Dysmyelopoiesis & 01 & 3.7 \\
Eosinophilia & 04 & 14.8 \\
Free floating cytoplasm & & \\
$\quad$ With LD bodies & 01 & 3.7 \\
$\quad$ Without LD bodies & 04 & 14.8 \\
$\begin{array}{l}\text { Intracellular LD bodies in cells other than } \\
\text { histiocytes (polymorph, metamyelocyte, and } \\
\text { megakaryocyte) }\end{array}$ & 02 & 7.4 \\
Bone marrow biopsy (total 19 cases) & & \\
Necrosis & & \\
Granuloma & 02 & 10.5 \\
\hline
\end{tabular}

It shows that fever was the most common presentation followed by hepatosplenomegaly. Tables 2, 3, and 4 show the common, uncommon, and atypical findings observed on peripheral blood smear, bone marrow aspirate cytology, and trephine biopsy examination in cases of leishmaniasis. 
TABLE 4: Atypical bone marrow findings observed in leishmaniasis.

\begin{tabular}{lcc}
\hline & $\begin{array}{c}\text { Number } \\
\text { of cases }\end{array}$ & $\begin{array}{c}\text { Percentage of } \\
\text { cases (\%) }\end{array}$ \\
\hline Bone marrow aspirate cytology & & \\
$\quad$ Aggregates of LD bodies & 03 & 11.1 \\
$\quad$ Kinetoplast only & 01 & 3.7 \\
$\quad$ Giant cells & 01 & 3.7 \\
Bone marrow biopsy (total 19 cases) & & \\
$\quad$ Increased fibrotic foci & 02 & 10.5 \\
\hline
\end{tabular}

Table 5 shows the comparison of hematological and bone marrow findings with previous studies.

\section{Discussion}

Leishmaniasis is considered to be endemic in focal areas in about 90 countries in tropics, subtropics, and Southern Europe while in India, 130 million population is at risk of the disease $[1,6]$. Visceral leishmaniasis is considered to be disease of low altitude as climatic and geographical factors play an important role in the distribution of vector, parasite, and reservoir [2, 7]. However, the present study showed that 21 cases were observed from the height of $500 \mathrm{~m}$ above sea level, and out of these 8 cases were from regions above $1500 \mathrm{~m}$ altitude, thus indicating an emerging focus of disease at higher altitudes. This may either be related to change in environmental factors, population migration, or invasion of the forests. Recently, some studies have also reported new focus of leishmaniasis at higher altitudes of Himalayan and sub-Himalayan regions of India [8-10]. Socioeconomic factors including illiteracy and poverty may be related to the spread of leishmaniasis as $66.6 \%$ of cases were present in lower economic group in our study. Fever and hepatosplenomegaly were the common clinical presentation in the present study which is consistent with other studies [11]. However the disease was clinically unsuspected in $81.4 \%$ of the cases primarily because its presence in nonendemic areas and secondarily as clinical features overlapped with other prevalent infections of this area such as malaria, enteric fever, and HIV or with liver diseases. This itself lays the importance of vigilant bone marrow aspirate examination for the search of LD bodies and observation of associated bone marrow cytology features even if the clinical suspicion is low. The present study observed that pancytopenia and thrombocytopenia were present in $96.2 \%$ cases which is in contrast with another study from endemic region which observed pancytopenia and thrombocytopenia in only $10 \%$ and $30 \%$ cases, respectively (Table 5) [4]. The probable reason may be delayed diagnosis due to late presentation of cases from remote areas in the study or association with other infections such as tuberculosis and typhoid. On bone marrow aspirate cytology, increased histiocytes and hemophagocytosis were an important common phenomenon observed in the present study (Figure 1) which is in contrast to previous study from Iran by Daneshbod et al. which has reported it to be uncommon cytological findings [4]. However another study
TABLE 5: Comparison of hematological and bone marrow findings of the present study with previous studies in leishmaniasis.

\begin{tabular}{|c|c|c|c|}
\hline & $\begin{array}{l}\text { Bhatia et al. } \\
\text { [3] } \\
\text { (\% of cases) }\end{array}$ & $\begin{array}{c}\text { Daneshbod } \\
\text { et al. [4] } \\
\text { (\% of cases) }\end{array}$ & $\begin{array}{c}\text { Present } \\
\text { study } \\
\text { (\% of cases) }\end{array}$ \\
\hline \multicolumn{4}{|l|}{ Peripheral blood smear } \\
\hline Pancytopenia & 75 & 10 & 96.2 \\
\hline Anemia & - & 70 & 100 \\
\hline Thrombocytopenia & - & 30 & 100 \\
\hline Leucopenia & - & 07 & 96.2 \\
\hline Monocytosis & 62 & - & 18.5 \\
\hline Nucleated RBC & 69 & - & 22.2 \\
\hline \multicolumn{4}{|l|}{$\begin{array}{l}\text { Bone marrow aspirate } \\
\text { cytology }\end{array}$} \\
\hline \multicolumn{4}{|l|}{ Distribution of LD bodies } \\
\hline Intrahistiocytic & 44 & - & 00 \\
\hline Extrahistiocytic & 31 & - & 3.7 \\
\hline Mixed & 25 & 69 & 96.2 \\
\hline Plasmacytosis & 56 & 77.9 & 96.2 \\
\hline Eosinophilia & - & 27.4 & 14.8 \\
\hline \multicolumn{4}{|l|}{ Free cytoplasmic bodies } \\
\hline With LD bodies & - & 31.3 & 3.7 \\
\hline Without LD bodies & - & 75.4 & 14.8 \\
\hline Granular bodies & - & 51.9 & 00 \\
\hline Erythroid hyperplasia & 38 & 78.9 & 81.4 \\
\hline Increased histiocytes & 81 & - & 100 \\
\hline Hemophagocytosis & 75 & 6.8 & 70.3 \\
\hline $\begin{array}{l}\text { Intracellular nonhistiocytic } \\
\text { LD bodies }\end{array}$ & 25 & 5.9 & 10.4 \\
\hline $\begin{array}{l}\text { Plasma cells with abnormal } \\
\text { inclusions }\end{array}$ & 38 & 2.4 & 14.8 \\
\hline Increased blasts & 06 & 01 & - \\
\hline Spore like organisms & - & 11.8 & - \\
\hline Aggregates of LD bodies & 56.2 & 15.7 & 11.1 \\
\hline Kinetoplast only & - & 8.8 & 3.7 \\
\hline Pseudo-Pelger-Huet & 25 & 4.9 & 00 \\
\hline Giant cells & - & 7.8 & 3.7 \\
\hline $\begin{array}{l}\text { Atypical histiocytes (Tart } \\
\text { cell, foam cells, and RS } \\
\text { cells) }\end{array}$ & 25 & 25.4 & 00 \\
\hline \multicolumn{4}{|l|}{ Bone marrow biopsy } \\
\hline Necrosis & 12 & 2.4 & 10.4 \\
\hline Granuloma & 68 & 23 & 5.2 \\
\hline Increased fibrotic foci & 19 & 4.4 & 10.4 \\
\hline Increased vascularity & 63 & 5.9 & 31.5 \\
\hline
\end{tabular}

has also reported hemophagocytosis as common finding which may be attributed to longer duration of symptoms [3]. Other associated features such as plasmacytosis, erythroid hyperplasia, plasma cells with abnormal inclusions (Russell bodies, Mott cells, and Crystals), and dysmyelopoiesis may 


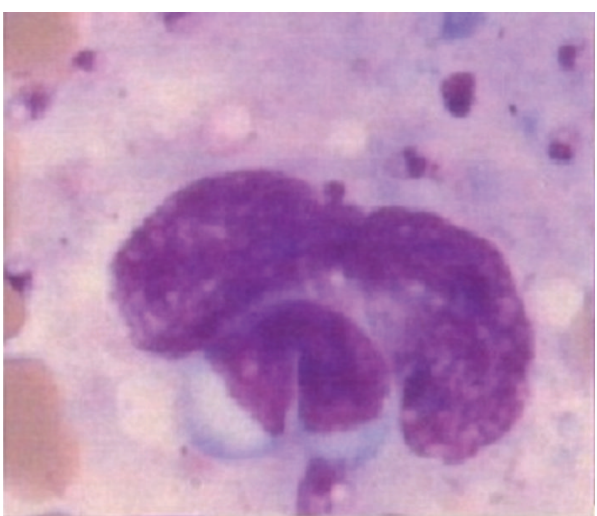

(a)

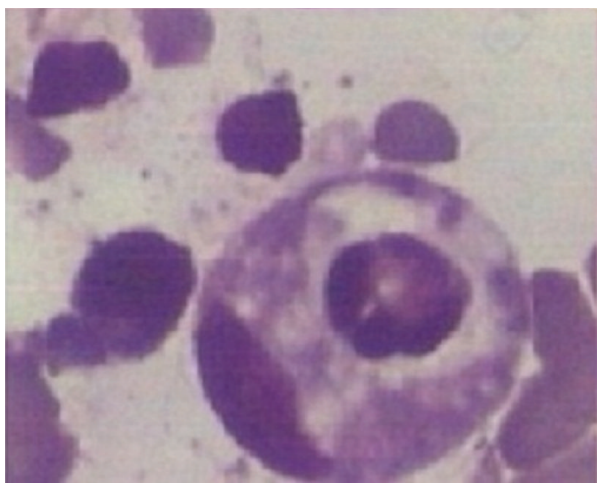

(c)

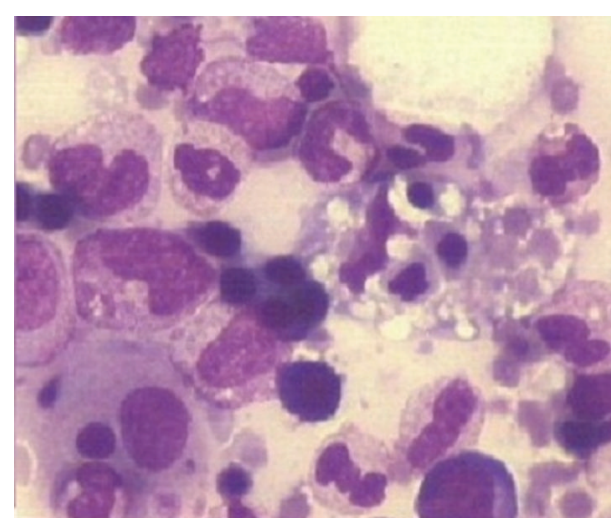

(b)

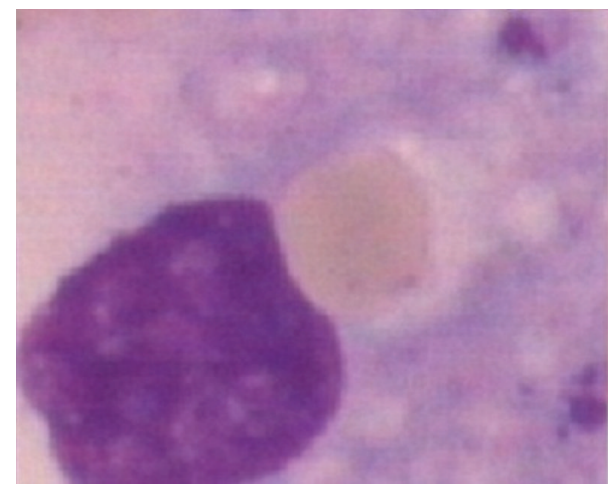

(d)

FIGURE 1: Bone marrow aspirate cytology showing hemophagocytosis in cases of leishmaniasis (Jenner Giemsa; ×400, ×1000).

also be helpful indicators of leishmaniasis (Figure 2). Aggregates of LD bodies in form of irregular flower shape, ring shape, and strap shape may also be uncommonly observed in leishmaniasis, which at times may mimic fungal spores or platelet aggregates and therefore necessitates a pathologist to have knowledge of such irregular aggregates (Figure 3). Another important difference that was observed from previous study in endemic region by Daneshbod et al. was lesser incidence of free cytoplasmic bodies and granular bodies in the present study (Table 5) [4]. Although the exact cause for this cannot be ascertained, the authors suggest that it may be related to APD which is higher in endemic regions leading to more cytoplasmic disintegration. The release of extracellular cysteine proteinase by amastigotes may be responsible for this cytoplasmic lysis [4]. The present study also indicates that LD body may also be present inside nonhistiocytic cells like polymorph, metamyelocyte, and therefore vigilant search of the parasite in nonhistiocytic cells should also be done (Figure 4). Interestingly, the LD body was also demonstrated in megakaryocyte and red blood cell in our study which has not been reported before (Figure 4). The bone marrow biopsy examination commonly indicates increased vascularity which may be due to reparative process and uncommonly shows necrosis or granuloma (Figure 2) which may be associated due to thrombosis of capillary lumen by parasites [12]. This may be supported by the fact that APD was more in cases showing necrosis on trephine biopsy examination.
Daneshbod et al. have observed granuloma (23\%) as common feature which is in contrast to the present study which observed granuloma (5.2\%) as uncommon feature and it may be attributed to decreased APD in nonendemic region [4]. Although giant cells, necrosis and granulomas are considered to be uncommon and atypical findings in VL in present study, their presence should also prompt close search of LD bodies on bone marrow aspirate cytology (Figure 2). This is especially important in areas were TB is endemic and may be associated with leishmaniasis.

\section{Conclusion}

Thus to conclude the knowledge of common, uncommon, and atypical features on bone marrow aspirate cytology is helpful in clinching an early and correct diagnosis of leishmaniasis especially in nonendemic areas where clinical suspicion is low. In contrast to previous study from endemic region, hemophagocytosis and pancytopenia were commonly observed while granular and free cytoplasmic bodies were uncommon morphological findings in the present study. The features will guide the pathologist for vigilant search of LD bodies in the marrow aspirate for definite diagnosis. In addition, it will also be useful in preventing the use of advanced and costly diagnostic modalities in the diagnosis of visceral leishmaniasis along with unnecessary workups. 


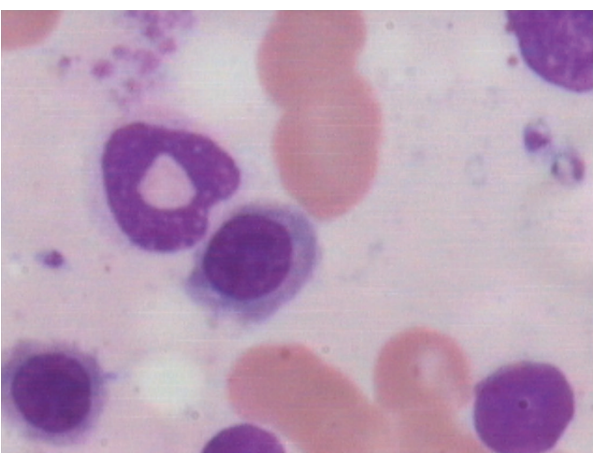

(a)

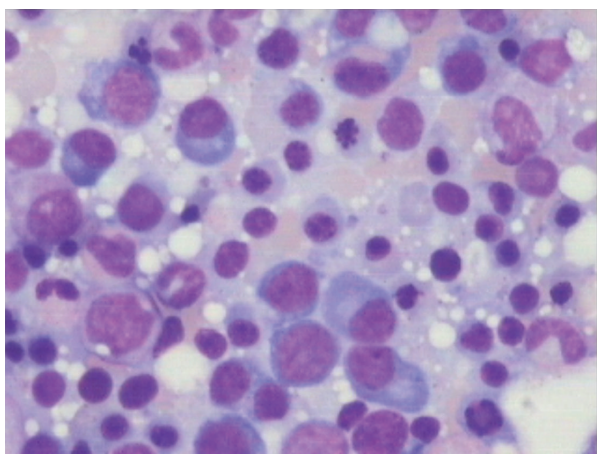

(c)

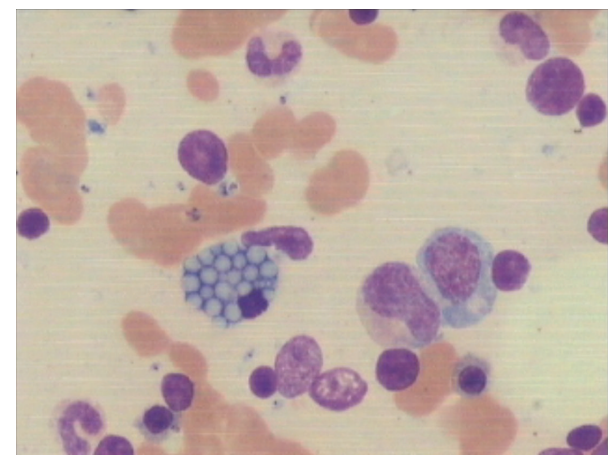

(b)

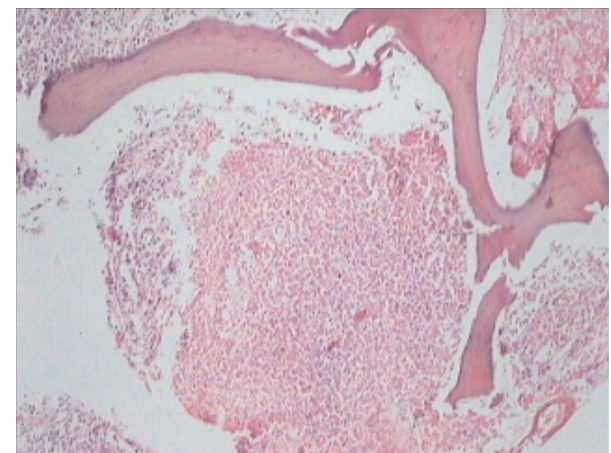

(d)

FIGURE 2: (a) bone marrow aspirate showing dysmyelopoiesis in a case of leishmaniasis, (b) bone marrow aspirate showing plasma cell with grape cell morphology and LD body, (c) bone marrow aspirate showing erythroid hyperplasia with increased plasma cells in leishmaniasis (Jenner Giemsa; $\times 1000$ ), and (d) bone marrow trephine biopsy showing granuloma and focal necrosis in a case of leishmaniasis (hematoxylin eosin; $\times 100)$.

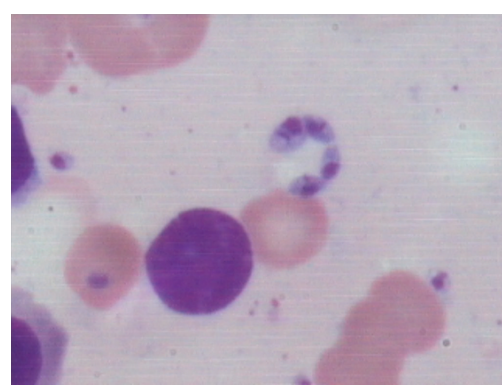

(a)

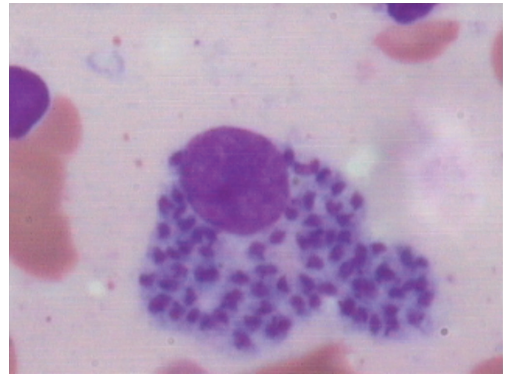

(d)

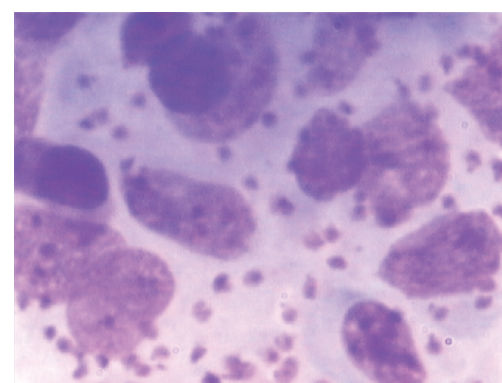

(b)

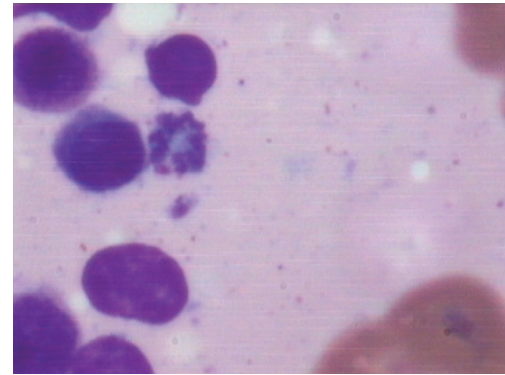

(e)

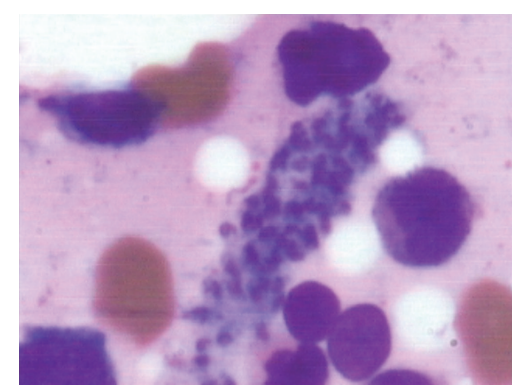

(c)

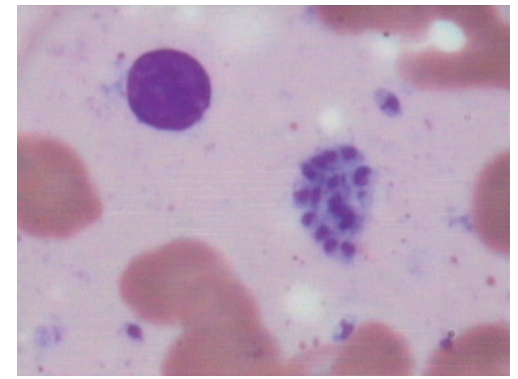

(f)

FIGURE 3: Bone marrow aspirate showing various forms of aggregates of LD bodies, (a) ring shaped aggregate of LD bodies, (b) intracellular and extracellular LD bodies, (c) strap shaped aggregate of LD bodies in cytoplasmic fragment, (d) intracellular aggregates of LD bodies, (e) floret arrangement of LD bodies and (f) LD bodies aggregate in cytoplasmic fragment (Jenner Giemsa; $\times 1000$ ). 


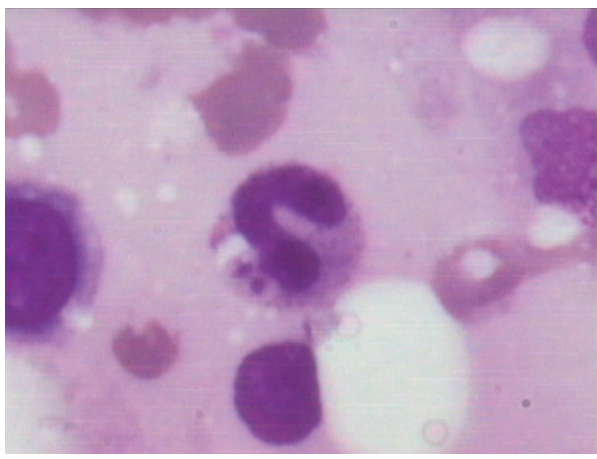

(a)

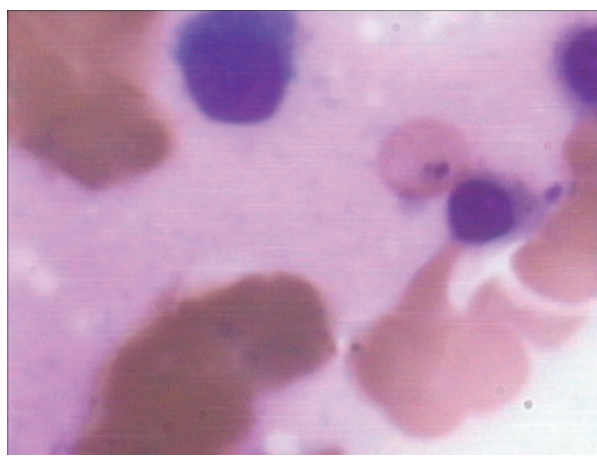

(c)

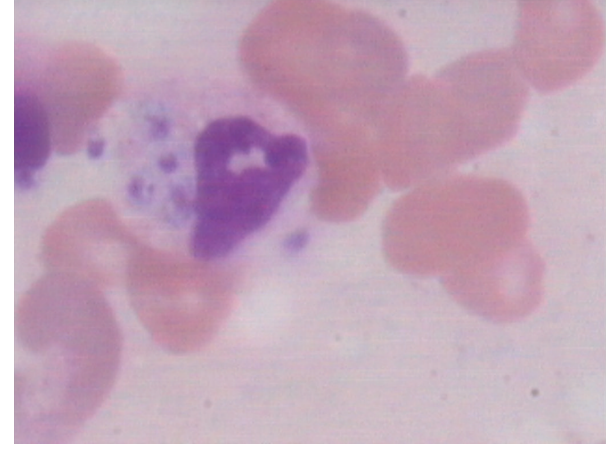

(b)

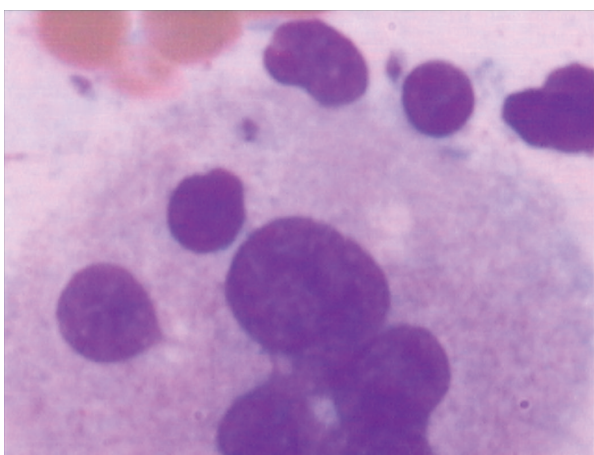

(d)

FIGURE 4: Bone marrow aspirate showing presence of LD bodies in nonhistiocytic cells, (a) LD body in metamyelocyte, (b) LD body in polymorph showing dysmyelopoiesis, (c) LD body in RBC and (d) LD body in megakaryocyte (Jenner Giemsa; $\times 1000$ ).

\section{Conflict of Interests}

The authors declare that they have no conflict of interests.

\section{References}

[1] B. L. Herwaldt, "Leishmaniasis," in Harrison's Principles of Internal Medicine, A. S. Fauci, E. Braunwald, D. L. Kasper et al., Eds., pp. 1296-1300, McGraw-Hill, New York, NY, USA, 17th edition, 2008.

[2] G. S. Bhunia, S. Kesari, A. Jeyaram, V. Kumar, and P. Das, "Influence of topography on the endemicity of Kala-azar: a study based on remote sensing and geographical information system," Geospatial Health, vol. 4, no. 2, pp. 155-165, 2010.

[3] P. Bhatia, D. Haldar, N. Verma, R. K. Marwaha, and S. Varma, "A case series highlighting the relative frequencies of the common uncommon and atypical/unusual hematological findings on bone marrow examination in cases of visceral leishmaniasis," Mediterranean Journal of Hematology and Infectious Diseases, vol. 3, article e2011035, 2011.

[4] Y. Daneshbod, S. J. Dehghani, and K. Daneshbod, "Bone marrow aspiration findings in kala-azar," Acta Cytologica, vol. 54, no. 1, pp. 12-24, 2010.

[5] K. K. Dhingra, P. Gupta, V. Saroha, N. Setia, N. Khurana, and T. Singh, "Morphological findings in bone marrow biopsy and aspirate smears of visceral kala azar: a review," Indian Journal of Pathology and Microbiology, vol. 53, no. 1, pp. 96-100, 2010.

[6] K. Park, "Leishmaniasis," in Park's Textbook of Preventive and Social Medicine, K. Park, Ed., pp. 279-282, M/s Banarsidas Bhanot, Jabalpur, India, 21st edition, 2011.
[7] D.-E. A. Elnaiem, J. Schorscher, A. Bendall et al., "Risk mapping of visceral leishmaniasis: the role of local variation in rainfall and altitude on the presence and incidence of kala-azar in eastern Sudan," American Journal of Tropical Medicine and Hygiene, vol. 68, no. 1, pp. 10-17, 2003.

[8] A. Joshi, A. Gulati, V. Pathak, and R. Bansal, "Post-Kala-Azardermal-leishmaniasis: an unusual presentation from Uttarachal (a non-endemic hilly region of India)," Indian Journal of Dermatology, Venereology and Leprology, vol. 68, no. 3, pp. 171173,2002

[9] R. Sharma, V. Mahajan, N. Sharma, and A. Sharma, "A new focus of cutaneous leishmaniasis in Himachal Pradesh (India)," Indian Journal of Dermatology, Venereology and Leprology, vol. 69, no. 2, pp. 170-172, 2003.

[10] S. K. Mahajan, P. Machhan, A. Kanga et al., "Kala-azar at high altitude," Journal of Communicable Diseases, vol. 36, no. 2, pp. 117-120, 2004.

[11] L. M. Prieto Tato, E. La Orden Izquierdo, S. Guillén Martn et al., "Visceral childhood leishmaniasis: diagnosis and treatment," Anales de Pediatria, vol. 72, no. 5, pp. 347-351, 2010.

[12] P. V. Kumar, M. Vasei, A. Sadeghipour et al., "Visceral leishmaniasis: bone marrow biopsy findings," Journal of Pediatric Hematology/Oncology, vol. 29, no. 2, pp. 77-80, 2007. 


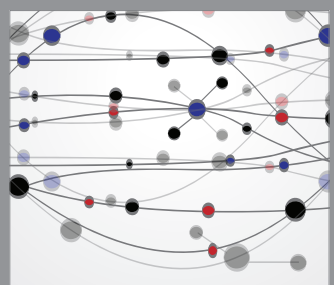

The Scientific World Journal
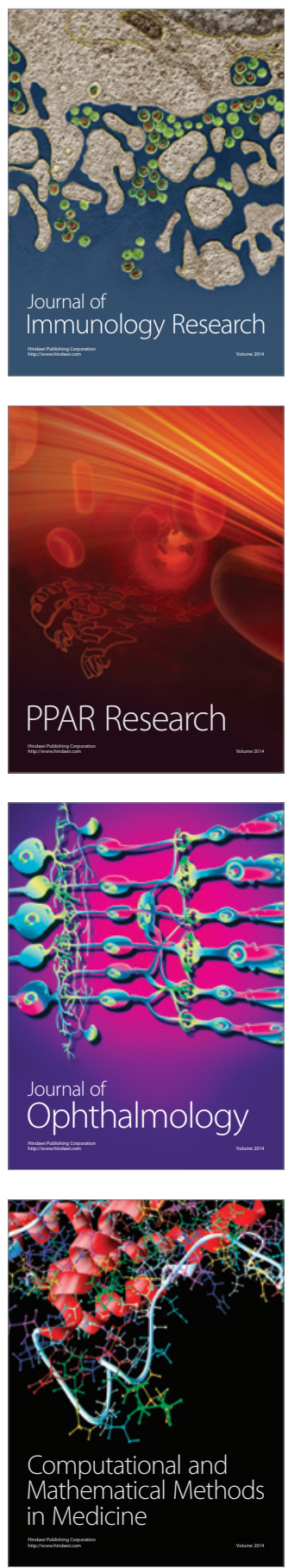

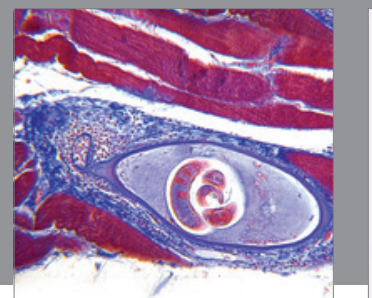

Gastroenterology

Research and Practice
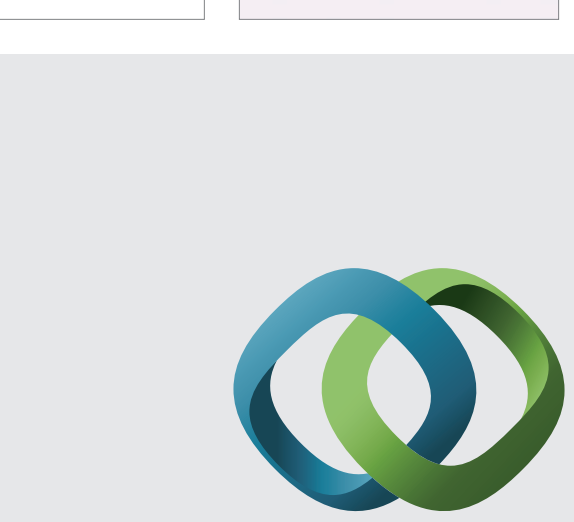

\section{Hindawi}

Submit your manuscripts at

http://www.hindawi.com
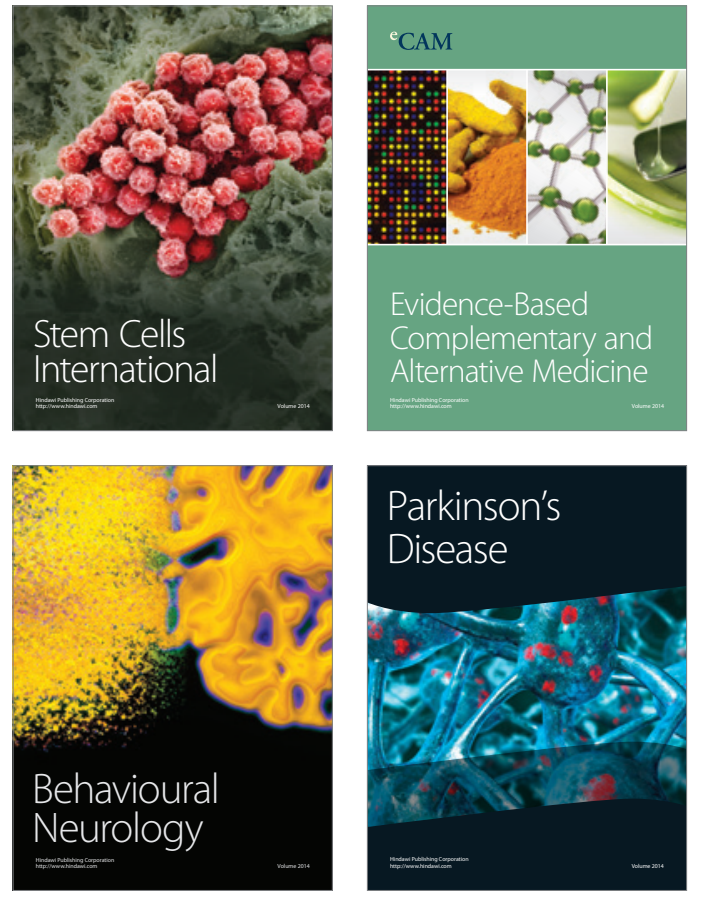
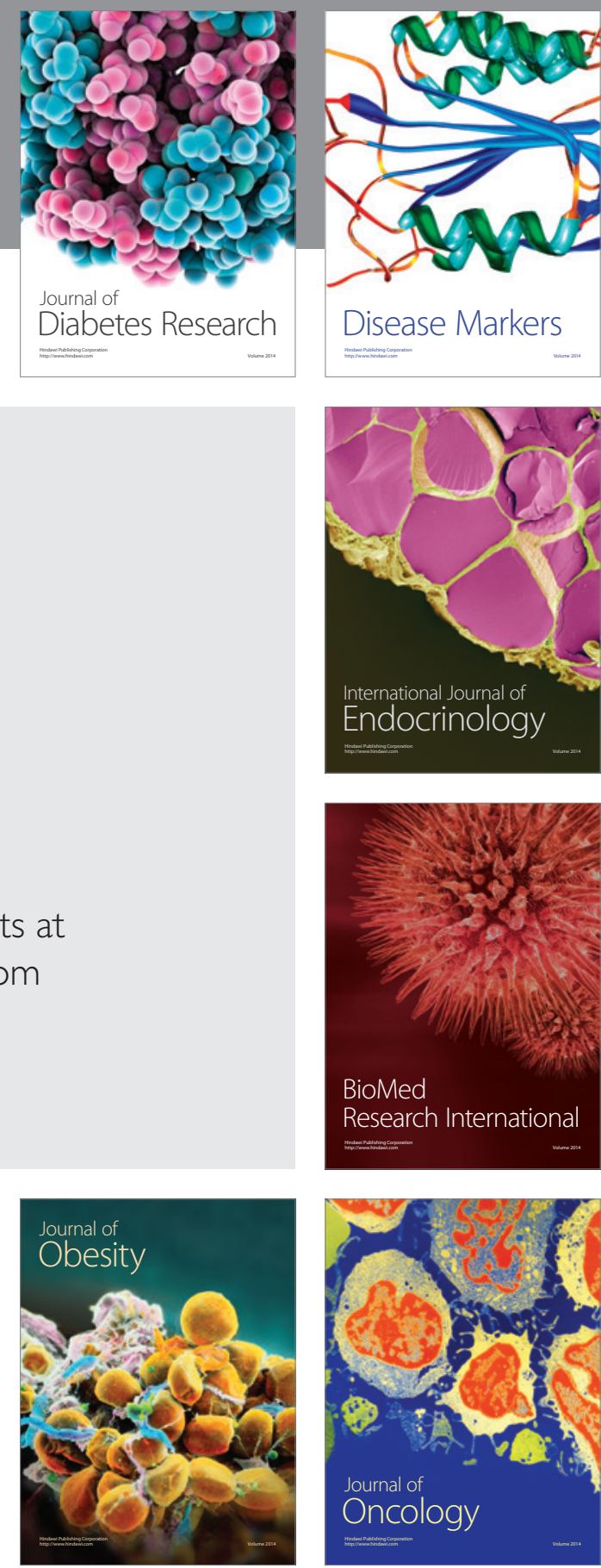

Disease Markers
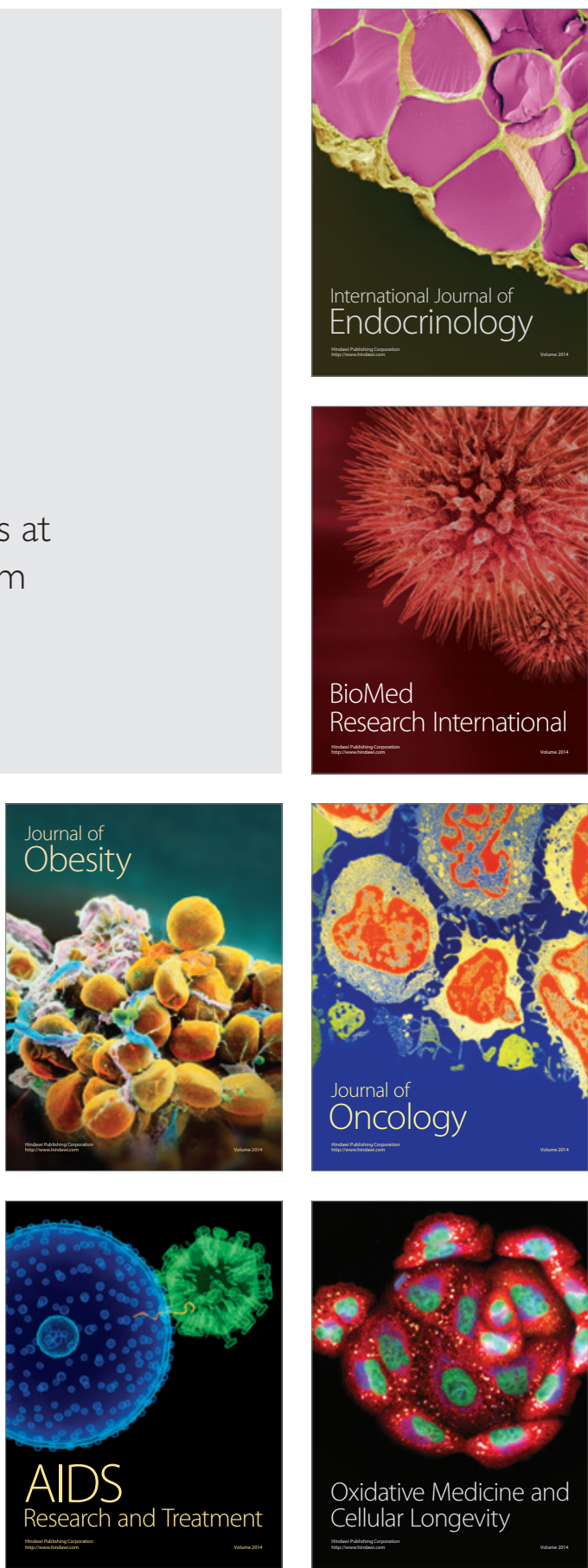